
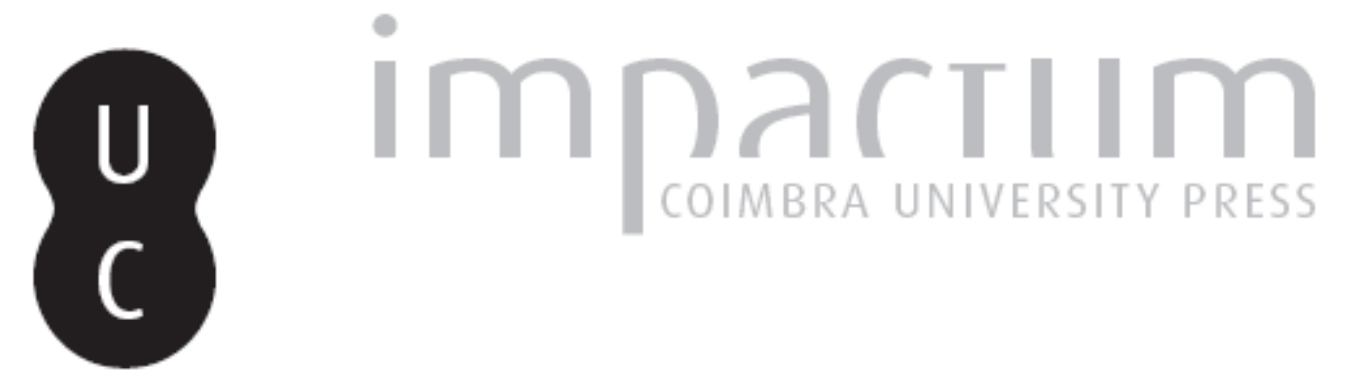

\title{
La asiriología según los asiriólogos
}

\section{Autor(es): Vita, JuanPablo}

Publicado por: Centro de História da Universidade de Lisboa

URL persistente:

URI:http://hdl.handle.net/10316.2/32965

DOI:

DOI:http://dx.doi.org/10.14195/0871-9527_22_1

Accessed : $\quad$ 26-Apr-2023 14:21:39

A navegação consulta e descarregamento dos títulos inseridos nas Bibliotecas Digitais UC Digitalis, UC Pombalina e UC Impactum, pressupõem a aceitação plena e sem reservas dos Termos e Condições de Uso destas Bibliotecas Digitais, disponíveis em https://digitalis.uc.pt/pt-pt/termos.

Conforme exposto nos referidos Termos e Condições de Uso, o descarregamento de títulos de acesso restrito requer uma licença válida de autorização devendo o utilizador aceder ao(s) documento(s) a partir de um endereço de IP da instituição detentora da supramencionada licença.

Ao utilizador é apenas permitido o descarregamento para uso pessoal, pelo que o emprego do(s) título(s) descarregado(s) para outro fim, designadamente comercial, carece de autorização do respetivo autor ou editor da obra.

Na medida em que todas as obras da UC Digitalis se encontram protegidas pelo Código do Direito de Autor e Direitos Conexos e demais legislação aplicável, toda a cópia, parcial ou total, deste documento, nos casos em que é legalmente admitida, deverá conter ou fazer-se acompanhar por este aviso.

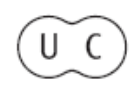



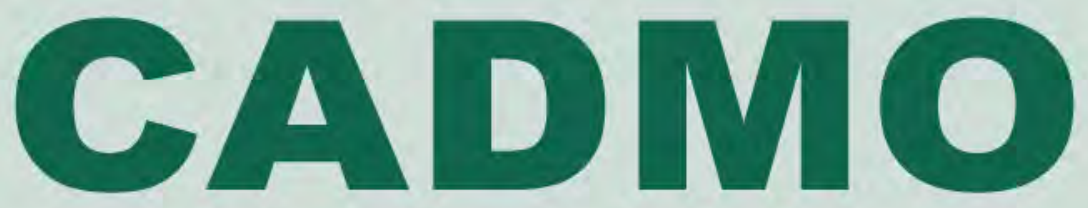

Revista de História Antiga

\author{
Centro de História \\ da Universidade de Lisboa
}

\title{
22
}

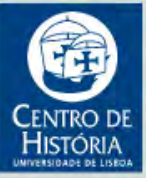

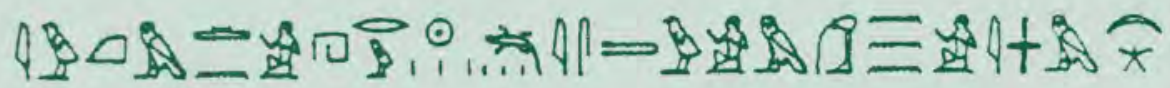

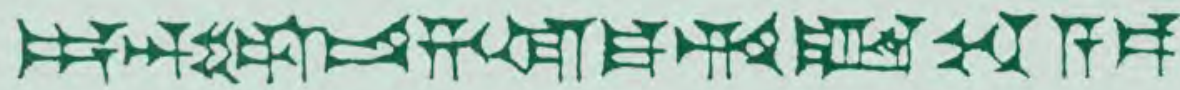
MHNIN AEI $\Delta \mathrm{E} \Theta \mathrm{EA} \Pi \mathrm{\Pi H} \Lambda \mathrm{HIA} \Delta \mathrm{E} \Omega$ 


\title{
LA ASIRIOLOGÍA SEGÚN LOS ASIRIÓLOGOS
}

\author{
JUAN-PABLO VITA \\ Consejo Superior de Investigaciones Científicas-CCHS, Madrid \\ jp.vita@csic.es
}

\section{Resumen}

A partir de la Segunda Guerra Mundial hizo su aparición un tipo de documento en el que el asiriólogo se dedica a reflexionar en primera acerca de la esencia misma de la asiriología: su razón de ser, sus objetivos, sus deberes, su utilidad, su futuro como ciencia. El presente artículo revisa las opiniones y reflexiones al respecto de destacados asiriólogos como Ephraim A. Speiser, Jean Bottéro, A. Leo Oppenheim, Paul Garelli, Stefan M. Maul, Aage Westenholz y Jean-Marie Durand.

Palabras-clave: Asiriología.

\begin{abstract}
After the Second World War a new type of document appeared which made Assyriologists reflect on the very essence of Assyriology: its raison d'être, its goals, its duties and usefulness, its future as a science. This paper reviews the opinions and reflections on this subject contributed by leading Assyriologists such as Ephraim A. Speiser, Jean Bottéro, A. Leo Oppenheim, Paul Garelli, Stefan M. Maul, Aage Westenholz and Jean-Marie Durand.
\end{abstract}

Key-words: Assyriology.

En 2007 se celebró el 150 aniversario del desciframiento oficial de la escritura cuneiforme mesopotámica ${ }^{1}$. En 1857, en efecto, la Royal Asiatic 
Society de Londres entregó a los ingleses E. Hincks, H. Rawlinson y F. Talbot, así como al francés J. Oppert, bajo sello y de manera independiente, una inscripción cuneiforme recién descubierta en Assur, con la petición de que entregaran su traducción del texto. Las traducciones fueron leídas el 25 de mayo de ese año. La comparación de las cuatro traducciones fue considerada lo suficientemente satisfactoria como para que la Society declarara como descifrada la escritura cuneiforme mesopotámica².

Menos de siete décadas después del desciframiento de la escritura cuneiforme, en fecha tan temprana como 1925, E. A. Wallis Budge (1857-1934), conservador del Museo Británico, trazó ya una historia de la asiriología en su libro The Raise and Progress of Assyriology (Londres). En el prefacio (pp. ix y xi) el autor expresa con claridad cuál es el principal objetivo de la obra: dejar bien establecido que la ciencia asiriológica fue fundada por el inglés H. C. Rawlinson, que fue apoyada y desarrollada de manera decisiva por el Museo Británico y que, en definitiva, el mundo le debe a Inglaterra la existencia y el establecimiento de la asiriología. De Inglaterra, la asiriología habría pasado a Alemania y a otros países europeos, y finalmente a los Estados Unidos de América. De esta manera, Wallis Budge recorre de manera pormenorizada la vida y obra de Rawlinson y expone la labor arqueológica, museológica y de edición de textos cuneiformes llevada a cabo por el Museo Británico. Sin embargo, a partir del capítulo XVI ( «The Spread of Assyriology») el autor amplía su objetivo inicial y mira también hacia otros países, ofreciendo breves semblanzas de asiriólogos de Francia, Suiza, Alemania, Italia, países escandinavos, Holanda y Estados Unidos de América. En el capítulo final («XVIII. Miscellaneous Observations») el autor cierra la obra presentando las razones que, en su opinión, fundamentan la importancia de la asiriología:

On it rests the foundation of a great deal of ancient history. It supplies unrivalled material for the study of early religion; and it has already revealed a world of social organization and material wellbeing at a very early period, of which the evidences elsewhere are partial and of doubtful explanation. Henceforth Assyriology must be reckoned as a necessary branch of humane learning; and in those scholastic institutions which are devoted to such subjects, Assyriology should be adequately represented.

En años más recientes, la historia de la asiriología ha recibido una atención creciente por parte de diversos autores. Cabe destacar en ese 
sentido los trabajos de Johannes Renger, durante años catedrático de Asiriología en la Universidad Libre de Berlín, en especial su «Die Geschichte der Altorientalistik und der vorderasiatischen Archäologie in Berlin von 1875 bis 1945», en W. Arenhövel y C. Schreiber (eds.), Berlin und die Antike, Berlin 1979, pp. 151-192, y la visión general que ofrece de la historia de esta disciplina en su artículo «Altorientalische Philologie und Geschichte», en M. Landfester (ed.), Der neue Pauly. Enziklopädie der Antike, vol. 13, pp. 101-114. El interés por la historia de la asiriología y, de manera más general, por la orientalística, se ha hecho especialmente patente en Alemania, con obras que exploran el papel desempeñado por personajes concretos en el desarrollo de diversas instituciones alemanas dedicadas al Próximo Oriente antiguo ${ }^{3}$ o que estudian la manera en la que los cambios políticos del país han afectado a las diversas disciplinas orientalísticas ${ }^{4}$.

La lista de trabajos que exploran aspectos diversos de la historia de nuestra disciplina puede ampliarse, con trabajos como los de, por ejemplo, Muazzez ÇiG, «Atatürk and the Beginnings of Cuneiform Studies in Turkey», Journal of Cuneiform Studies 40 (1988) pp. 211-216, o Jerrold S. Cooper, «Posing the Sumerian Question: Race and Scholarship in the Early History of Assyriology», Aula Orientalis 9 (1991) pp. 47-66. Este interés de carácter historiográfico también se encuentra en disciplinas afines como la Hititología, con obras como el catálogo colectivo From Bogazköy to Karatepe. Hittitology and the Discovery of the Hittite World (Nisan - Istanbul 2001) y el libro de Robert Oberheid, Emil O. Forrer und die Anfänge der Hethitologie: eine wissenschaftshistorische Biografie, Berlin $2007^{5}$. El lector interesado en la vida y obra de los principales orientalistas de las últimas décadas leerá también con provecho las secciones «Dem Gedächtnis der Toten» y «Kurze Worte des Gedenkes» de la revista Archiv für Orientforschung.

Algunos asiriólogos han producido también documentos de otra naturaleza acerca de su disciplina. Así, Donald J. Wiseman, profesor en la School of Oriental and African Studies de la Universidad de Londres, redactó en 1967 un documento titulado La recherche en Europe: Assyriologie (Estrasburgo $)^{6}$, para el entonces «Conseil de la coopération culturelle» del «Conseil de l'Europe»?. El documento se estructura en los capítulos siguientes: I. Introduction. Domaine des études d'assyriologie, II. Méthode d'enquête, III. Institutions d'enseignement, IV. Recherche, V. Publications, VI. Coopération internationale, VII. Instituts européens du Proche-Orient, 
VIII. Recommandations, seguido de una serie de anexos. En el capítulo III (p. 9) se señala la existencia en España de catorce universidades, de las cuales sólo en una era la asiriología materia de enseñanza, y ésta por medio de un solo especialista; más adelante (p. 22) se especifica que se trata en realidad del antiguo «Instituto Arias Montano de Estudios Hebraicos» del Consejo Superior de Investigaciones Científicas (CSIC), en Madrid, y que el especialista en cuestión era el Dr. J. M. Peñuela (p. 31) ${ }^{8}$. También se señala la existencia en España, en el Monasterio de Montserrat (p. 13), de una colección importante y representativa de documentos cuneiformes y de objetos arqueológicos próximo-orientales ${ }^{9}$.

Otro género, que cuenta aún con muy pocos títulos, es el de las autobiografías de orientalistas. Los ejemplos más representativos al respecto tal vez sean los libros de Samuel Noah Kramer (1897-1990), eminente sumerólogo del Oriental Institute de Chicago ${ }^{10}$, titulado In the World of Sumer. An Autobiography (Detroit 1986) ${ }^{11}$, y de Cyrus H. Gordon (1908-2001), profesor en diversas universidades estadounidenses, $A$ Scholar's Odyssey (Atlanta 2000). Una variante del género autobiográfico son las entrevistas efectuadas, por diversos motivos, a orientalistas. Representativa en este sentido es la que ofrece Michael C. Astour, en la obra-homenaje que le dedican G. D. Young, M. W. Chavalas y R. E. Averbeck (eds.), Crossing Boundaries and Linking Horizons, Bethesda 1997 (pp. 1-36: «An Interview with Michael Astour»), en la que el homenajeado rememora sus años de estudiante, recuerda los maestros que más le han influido, comenta algunas de sus principales obras y subraya la importancia de los estudios de geografía histórica, campo en el que destacó especialmente.

A partir de la Segunda Guerra Mundial, y con cierta regularidad hasta nuestros días, hizo su aparición un tipo de documento en el que el asiriólogo se dedica a reflexionar en primera persona no ya acerca de su propia vida o la historia de su disciplina, sino acerca de la esencia misma de la asiriología: su razón de ser, sus objetivos, sus deberes, su utilidad, su futuro como ciencia. El más antiguo del que tenemos noticia, titulado "Oriental Studies and Society", se debe a Ephraim A. Speiser (1902-1965), profesor en la Universidad de Pensilvania. El texto fue publicado en 1946 en la revista americana Journal of the American Oriental Society 66 , pp. 193-19712. Para comprender mejor las ideas expresadas por el autor, conviene señalar que durante la Segunda Guerra Mundial, Speiser estuvo al frente de la «Near East section, research and analysis branch, 
of the Office of Strategic Services» del ejército de los Estados Unidos de América, y que este artículo fue publicado inmediatamente después de la contienda. Su experiencia personal le lleva a proponer una visión muy práctica, muy aplicada, de los estudios orientales, haciendo mucho hincapié en la posición del orientalista como científico y como ciudadano en general. La Segunda Guerra Mundial habría sacado a los orientalistas de su anonimato, y la realidad de la política contemporánea debe llevar a éstos a abandonar su «torre de marfil» y aceptar su obligación de poner sus conocimientos al servicio de la sociedad ${ }^{13}$ así como avanzar hacia una mayor interdisciplinariedad con otras disciplinas más o menos afines. Las razones principales serían que el Oriente en general (Oriente Próximo, India, China, etc.) se ha convertido en un área vital para las sociedades occidentales por razones demográficas, políticas y económicas, y que el Oriente Próximo moderno hunde profundamente sus raíces en la Antigüedad, de manera que «The modern Orient is a product of its past probably in a more intimate sense that any other part of the world. We cannot hope to understand it if we arbitrarily splinter off the present from its antecedents» (p. 581); "basic research about the rest of the world is one of the preliminaries to an effective foreign policy» (p. 583).

Una posición diametralmente opuesta a la de Speiser sería la expresada por el eminente asiriólogo francés Jean Bottéro (1914-2007), profesor en la École Pratique des Hautes Études (París), en su artículo «Eloge d'une science inutile: l'orientalisme», publicado en 1982 en la revista Akkadica 30 , pp. $12-26^{14}$. Se trata del texto de una conferencia pronunciada por el autor con motivo del cincuenta aniversario de la fundación del Instituto de Filología y de Historia orientales de la Universidad Libre de Bruselas. Según Bottéro, la asiriología lo habría neutralizado como persona y como ciudadano: «la discipline à laquelle je me suis voué, en me tournant exclusivement vers le passé, m'a, comme tel, rendu incapable d'intervenir dans la vie de mes contemporains» (p. 14). Los orientalistas se encontrarían entre los sabios que, por dirigir su curiosidad hacia los orígenes de la historia humana, dejarían en paz al universo y a sus habitantes: «Leur discipline acquiert, de ce fait, une utilité négative» (p. 15). A pesar de todo, sí que cabría descubrirle a la asiriología un aspecto útil, en relación con la propia identidad histórica de la civilización occidental (pp. 23-24):

Nous voici...tributaires lointains des Sumériens et des Babyloniens, lesquels constituent donc nos plus vieux ancêtres discernables en ligne ascendante directe. De loin, de très loin, ils sont de notre famille, ils 
font partie de notre passé... si nous voulons le comprendre sur le plan génétique, si nous voulons retrouver nos ancêtres, faire le bilan de la partie la plus ancienne et foncière de notre héritage, et rechercher de notre devenir cette explication... que seule peut nous fournir l'Histoire, nous devons remonter jusqu'à ces Mésopotamiens archaïques, tout au bout de notre horizon... Voilà comment j'ai renoncé à imputer à I'Assyriologie une inutilité totale... j'ai appris à la tenir, non seulement pour utile, mais (objectivement!) indispensable à une juste et globale compréhension de notre propre histoire... elle est à notre service pour nous fournir... nos plus vieux papiers de famille pour couronner notre passé, inaugurer notre génèse, et nous conduire jusqu'à la source première de cet énorme fleuve qui nous porte toujours.

Bottéro, en realidad, se expresa en la misma línea de pensamiento que ya había trazado con anterioridad A. Leo Oppenheim (1904-1974), profesor también en el Oriental Institute de Chicago. Su célebre obra Ancient Mesopotamia. Portrait of a Dead Civilization (Chicago 1964) ${ }^{15}$ viene precedida por una introducción que lleva por título «Assyriology: Why and How?» (pp. 7-30), texto publicado con anterioridad en la revista Current Anthropology 1 (1959) pp. 409-423. Tras hacer un repaso al tipo de disciplinas que se ocupan del Próximo Oriente antiguo y a los documentos que sirven de base al estudio de su historia y de sus lenguas, el autor señala la excesiva especialización y parcelación de subcampos de investigación como uno de los males de esta disciplina, señalando la interdisciplinariedad, la colaboración con especialistas de otras áreas que, además, también se encuentran representadas en los textos cuneiformes, como posibles remedios a esta situación (pp. 28-29). Finalmente señala cuál es, en su opinión, la función esencial del asiriólogo (p. 30):

No history of science and technology that claims scholarly status can be written when its author has to rely on inadequate translations of cuneiform texts pertaining to his subject. The Assyriologist should become aware that he holds the keys to a potential wealth of information covering more than two millennia of one of the great civilizations. If he is in need of a raison d'être, here it is ${ }^{16}$.

Las ideas de Oppenheim encontraron pronto un eco explícito en el libro L'assyriologie (Paris 1964) de Paul Garelli (1924-2006), profesor en el Collège de France (véanse en especial las páginas 35-40 y 119-126). Tras señalar que «c'est finalement toujours la même question qui guidera les 
recherches: quels étaient ces hommes que nous avons la chance de saisir au seuil de la préhistoire, et que nous apprennent-ils sur nous-mêmes? » (p. 40), el autor da una definición de lo que, en su opinión, es la figura del asiriólogo: «Ebauches, univers en gestation, telle est la dominante de I'histoire mésopotamienne, et c'est dans cette perspective que doivent être menées les recherches ... L'assyriologue est fondamentalement l'homme des genèses» (p. 119). El asiriólogo también tendría una influencia en el mundo actual a través de la esfera religiosa (p. 120):

Dans la mesure ou l'assyriologue conditionne le développement des études bibliques, elle exerce une influence sur deux des principales religions actuellement vivantes: le judaïsme et le christianisme. La découverte des textes cunéiformes a profondement modifié la position des exégètes et des théologiens, et la répercussion commence à s'en faire sentir dans les rangs des autres fidèles. S'il est un point par lequel l'assyriologue a pu marquer le monde contemporain, c'est celui-là.

El interés por las posibles funciones, deberes y objetivos de la Asiriología y del asiriólogo ha continuado hasta nuestros días. Destaca en este sentido el discurso pronunciado en 1998 por Stefan M. Maul, profesor de Asiriología en la Universidad de Heidelberg, con motivo del centenario de la Deutsche Orient-Gesellschaft. El discurso fue publicado en ese mismo año en la revista Mitteilungen der Deutschen Orient-Gesellschaft 130, pp. 266-274, con el título «Wiederstehende Welten. Aufgaben und Möglichkeiten moderner Altorientalistik». Según el autor, el desciframiento y la edición de textos cuneiformes seguirán situándose en el futuro en el centro de la actividad de esta disciplina, aunque el centro de gravedad de la investigación se irá desplazando cada vez en mayor medida hacia la investigación de los propios textos (p. 271). En consecuencia (pp. 271-272):

Die Altorientalistik wird in Zukunft mit ihren Ergebnissen wieder mehr in die breite Öffentlichkeit treten ... Es wird vielmehr Aufgabe des Faches sein, den Wissenschaften und dem kulturellen Bewußtsein unserer Gesellschaft zu erschließen, von welch immenser historischer und kulturhistorischer Bedeutung Texte und Hinterlassenschaften des Alten Orients sind ... Die altorientalischen Quellen sind hierbei nicht etwa eine exotische Bereicherung, sondern wesentlicher Natur, da die altorientalischen Kulturen durch ihren großen Einfluß auf die griechisch-römische und christlich-jüdische Kultur in ihren Auswirkungen Weltbild, Religion und Wissenschaften der Gegenwart entscheidend geprägt haben. Darüber hinaus kann die Auswertung von Texten, wie im Falle 
der Erforschung altorientalischer landwirtschaftlicher Methoden, sogar für die Gegenwart von unmittelbar greifbarem Nutze sein.

Maul añade igualmente que la enseñanza de la Orientalística antigua no debería faltar en ninguna Universidad que aprecie la dimensión histórica de las disciplinas que en ella se imparten (p. 272).

¿Qué futuro puede depararle a la Asiriología nuestra sociedad actual? Es la cuestión que se ha planteado recientemente el asiriólogo danés Aage Westenholz (Universidad de Copenhague), en su artículo «Does Assyriology have a Future?", publicado en la revista KASKAL. Rivista di storia, ambienti e culture del Vicino Oriente Antico 3 (2006) pp. 275-283. La asiriología sale bastante mal parada de las reflexiones y consideraciones de este especialista. Considera, por ejemplo, que se trata de una de las ramas más esotéricas de las Humanidades, "one that endeavors to explore a long dead and forgotten culture only distantly related to our own" (p. 276). Los asiriólogos se habrían convertido en especialistas que sólo se comunican entre ellos, sin que exista más público que esté interesado en sus trabajos. Los políticos desdeñan este tipo de estudios por resultar «no rentables» y los gobiernos siguen tolerando la asiriología sólo porque todavía se consideran protectores de la cultura. La asiriología sólo sería hoy en día "a charming relic of nineteenth-century culture, tolerated because of cultural inertia in politics and because it costs so little» (p. 278). Se pregunta, en definitiva, que por qué razones deberían nuestras sociedades seguir mantener a los especialistas que se dedican a la historia del Próximo Oriente antiguo. Finalmente, regresa a la pregunta que da título a su artículo, para dar una respuesta negativa: «So, does Assyriology have a future? As I have enumerated the facts so far, it doesn't» (p. 282). Sin embargo, como si quisiera suavizar la dureza de su conclusión, termina sus consideraciones abriendo alguna posibilidad de futuro para la disciplina, en la medida en que aún somos necesarios para las necesidades culturales, incluso existenciales, de una pequeña proporción de personas, de manera que seguimos manteniendo una responsabilidad social. El artículo se cierra planteándose de nuevo la pregunta: «does Assyriology have a future? - I think this is within our powers to give one, if we want to. Perhaps even politicians may get to appreciate us».

Afortunadamente, más optimista que Westenholz respecto al futuro de nuestra disciplina se mostró Jean-Marie Durand, profesor en el Collège de France (Cátedra de Asiriología), en su lección inaugural pronunciada 
en 1999 y publicada en el año 2000 por el Collège de France en París: "C'est justement cet intérêt des étudiants pour des matières auxquelles rien ne prédispose dans notre système éducatif ou culturel qui fait penser que la discipline n'est pas encore prêt de s'éteindre» (p. 6).

\section{Notas}

(1) Sirvan las líneas que siguen de modesto homenaje de admiración, amistad y cariño al Profesor Don Antonino González Blanco (Universidad de Murcia).

(2) El lector interesado encontrará una descripción actualizada, didáctica y bien ilustrada de esta historia en la obra colectiva editada por Brigitte LION y Cécile MICHEL, Les écritures cunéiformes et leur déchiffrement, París, 2007.

(3) Véanse en especial las diversas contribuciones de Olaf Matthes, como por ejemplo «Eduard Meyer und die Deutsche Orient-Gesellschaft», Mitteilungen der Deutschen Orient-Gesellschaft 128 (1996), pp. 173-218; id., James Simon: Mäzen im Wilheminischen Zeitalter, Berlin, 2000.

(4) Véase al respecto en especial la obra editada por W.-H. KRAUTH y R. WOLZ, Wissenschaft und Wiedervereinigung. Asien- und Afrikawissenschaften im Umbruch, Berlin, 1998, recensionada por Peter Heine, «Orientalistik vor und nach der Wende», Orientalistische Literaturzeitung 94 (1999) cols. 434-446, y la más reciente de J. ELVERT y J. NIELSEN-SIKORA (eds.), Kulturwissenschaften und Nationalsozialismus, Wiesbaden 2008, en especial el capítulo de Johannes Renger, «Altorientalistik», pp. 469-502.

(5) La obra contiene en apéndice, pp. 349-410, un buen número de breves pero documentadas biografías de orientalistas.

(6) D. J. Wiseman es también el autor de «The Expansion of Assyrian Studies: An Inaugural Lecture», School of Oriental and African Studies, University of London, 1962, documento que no nos ha sido accesible.

(7) El informe se enmarcaba dentro de la siguiente política europea, descrita en el prefacio: «Le groupe d'étude sur les moyens d'une coopération organique entre les universités européennes a proposé la mise en chantier de travaux pilotes, sous forme de rapports analytiques portant sur l'état de la recherche dans différents domaines du savoir ou spécialités, qui indiqueraient les ressources dont disposent les pays membres du Conseil de la coopération culturelle, l'orientation qu'y prend la recherche et les besoins qu'ils éprouvent en la matière».

(8) Acerca de la contribución española al descubrimiento y estudio de la Historia y la Arqueología del Próximo Oriente antiguo véase J. M ${ }^{a}$ CÓRDOBA y $\mathrm{M}^{\mathrm{a}} \mathrm{C}$. PÉREZ DÍE (eds.), La aventura española en Oriente (1166-2006), Madrid, 2006.

(9) Textos cuneiformes que han sido editados en gran parte por Manuel Molina (CSIC-CCHS), en sus obras Tablillas administrativas neosumerias de la Abadía de Montserrat (Barcelona). Copias cuneiformes, Roma, 1993, y Tablillas administrativas neosumerias del Museo de Montserrat. Transliteraciones e índices, Barcelona, 1996.

(10) Autor bien conocido también fuera de los círculos académicos gracias a su popular obra de divulgación History Begins at Sumer: Thirty-Nine Firsts in Man's Recorded History, University of Pennsylvania Press, 1956 (edición española: La historia empieza en Sumer, Barcelona, 1958 [ed. Aymà] y ed. Orbis, 1985). 
(11) El lector interesado leerá con provecho las valoraciones que hacen del libro tanto Miguel Civil (en el prefacio al libro) como Cyrus H. Gordon en una reseña en el Journal of Cuneiform Studies 39 (1987), pp. 247-250.

(12) Republicado en el volumen de J. J. FINKELSTEIN y M. GREENBERG (eds.), Oriental and Biblical Studies. Collected Writings of E. A. Speiser, Philadelphia, 1967, pp. 573-585.

(13) «It should be our aim, as scholars and citizens, to make our study of the Orient as comprehensive as possible, without encroaching on alien, not to say hostile, fields», p. 577.

(14) Republicado en su obra Méspotamie. L'écriture, la raison et les dieux, Paris, 1987, pp. 43-61.

(15) Libro editado en español como La Antigua Mesopotamia. Retrato de una civilización extinguida, Gredos, Madrid, 2003 (traducción: Ignacio Márquez Rowe).

(16) En nota a pie de la página 30, Oppenheim señala: «For a critical review of the attitude expressed in this section ("Assyriology- Why and How?"), the reader is referred to D. J. Wiseman, The Expansion of Assyrian Studies: An Inaugural Lecture (School of Oriental and African Studies, University of London, 1962)». 